\title{
Mexique : recherche et politique en éducation
}

\section{Norma Georgina Gutiérrez}

Traducteur : Odile Luginbühl

\section{OpenEdition}

\section{Journals}

Édition électronique

URL : http://journals.openedition.org/ries/2140

DOI : 10.4000/ries. 2140

ISSN : 2261-4265

Éditeur

Centre international d'études pédagogiques

Édition imprimée

Date de publication : 1 décembre 2011

Pagination : 21-28

ISBN : 978-2-85420-593-0

ISSN : $1254-4590$

\section{Référence électronique}

Norma Georgina Gutiérrez, « Mexique : recherche et politique en éducation », Revue internationale d'éducation de Sèvres [En ligne], 58 | décembre 2011, mis en ligne le 01 décembre 2014, consulté le 01 mai 2019. URL : http://journals.openedition.org/ries/2140 ; DOI : 10.4000/ries.2140

Ce document a été généré automatiquement le 1 mai 2019.

(c) Tous droits réservés 


\title{
Mexique : recherche et politique en éducation ${ }^{1}$
}

\author{
Norma Georgina Gutiérrez Serrano
}

\section{Structure, chiffres, programmes}

\section{Le droit à l'éducation dans un pays multiculturel}

1 Les États-Unis du Mexique sont un pays d'Amérique du Nord d'une superficie de 1964375 $\mathrm{km}^{2}$, avec un statut de république fédérale comprenant 28 États et un district fédéral. Le recensement de 2010 dénombre une population de 112 millions de Mexicains, dont 48,8\% d'hommes et $51,2 \%$ de femmes. L'espagnol est la langue principale, mais on compte 67 autres langues indigènes encore parlées.

2 La diversité linguistique est le reflet d'une grande diversité culturelle qui vient seulement d'être reconnue, alors que le Mexique a proclamé depuis 1810 son indépendance par rapport à l'Espagne et que la Constitution actuelle réaffirme le caractère démocratique de l'organisation politique et sociale du Mexique en tant que république fédérale.

3 Le système éducatif mexicain (SEM) se fonde sur la Constitution politique des États-Unis du Mexique, qui inscrit le droit à l'éducation dans ses articles 3 et 31 et précise que l'enseignement assuré sous la responsabilité de l'État doit être laïc et gratuit. Elle fixe également le caractère obligatoire de l'éducation de base, qui compte actuellement trois années de préscolaire, six d'enseignement primaire et trois de secondaire.

4 Les bases de l'enseignement public au Mexique ont été mises en place au fur et à mesure des grands mouvements nationaux du XIXe siècle, qui culminèrent en 1910, avec la première révolution du XXe siècle. En 1921, le gouvernement fédéral a créé le secrétariat à l'Éducation publique, instance officielle qui a régi l'organisation, la structure, le développement et la dynamique du SEM tout au long de ses quatre-vingt-dix années d'existence. Plusieurs grandes figures d'intellectuels, comme José Vasconcelos, Narciso Bassols, Plutarco Elias Calles et Jaime Torres Bodet, ont été en charge de ce secrétariat. 
Sous leur responsabilité, des politiques ambitieuses et de grande portée sociale ont été conduites. L'éducation a pris alors une grande importance dans la politique nationale mexicaine, au point qu'un de ses principaux protagonistes, Jaime Torres Bodet, fut nommé directeur général de l'Unesco en 1948.

\section{Une structure complexe, un taux de scolarisation mal défini}

Depuis les premières décennies du $\mathrm{XX}^{\mathrm{e}}$ siècle, le système éducatif mexicain s'est développé, mais aussi complexifié et diversifié, et il a connu de nombreuses réformes.

Globalement, le système éducatif compte actuellement trois grands cycles d'enseignement, chacun regroupant différents niveaux: enseignement de base (préscolaire, primaire et secondaire); enseignement moyen supérieur (professionnel, technique et baccalauréat); enseignement supérieur (technique supérieur, licence et troisième cycle). L'enseignement supérieur se partage entre les universités, les instituts, les centres technologiques et les écoles normales, celles-ci étant destinées, depuis le XIX ${ }^{\mathrm{e}}$ siècle, à former les futurs enseignants.

7 L'ensemble du système accueille environ 34,4 millions d'élèves et étudiants, selon les dernières estimations officielles (Calderon, 2011). On estime que 88 \% d'entre eux relèvent $\mathrm{du}$ secteur public, c'est-à-dire avec prise en charge du gouvernement, et $12 \%$ d'établissements privés ou spécialisés.

Par niveau, la population scolaire se répartit approximativement ainsi :

- enseignement de base : plus de 25,5 millions d'élèves (75,5\% de la population scolaire) ;

- enseignement moyen supérieur : plus de 4 millions d'élèves ( $12 \%$ de la population scolaire) ;

- enseignement supérieur: un peu plus de 2,8 millions d'étudiants (à peine 8,4\% de la population scolaire).

9 À la fin des années quatre-vingt, le discours politique tendait à affirmer que l'enseignement primaire était parvenu à un taux de scolarisation " universel», touchant tous les enfants concernés, puis on a parlé d'une scolarisation « quasi universelle » et, actuellement, on dit avoir atteint de « larges » franges de scolarisation - ce qui manifeste plus de précautions quant à la formulation du bilan de l'enseignement primaire. Quant à l'enseignement supérieur, on observe à l'évidence, même dans les peu fiables chiffres officiels, une notoire et dramatique chute du taux de scolarisation.

10 De manière générale, les évaluations présentées par le gouvernement fédéral dans un récent rapport font apparaitre de fortes divergences quant au taux de scolarisation, dans sa totalité et par niveau d'enseignement. Ces divergences apparaissent entre spécialistes de l'éducation, surtout pour l'enseignement supérieur, mais aussi entre différents services, à l'intérieur de la même structure gouvernementale.

11 Par exemple, pour ce qui relève du taux de scolarisation dans l'enseignement moyen, le secrétariat à l'Éducation publique affirme que l'on atteint un taux de $66 \%$ de la tranche d'âge correspondant à ce niveau d'études, alors que l'Institut national d'évaluation en éducation (INEE) n'en compte que $52 \%$, ce qui correspond à une différence de 14 points entre les deux chiffres (INEE, 2009). Il en résulte de grands doutes sur les estimations fournies, même par des services officiels.

12 En ce qui concerne le corps enseignant, on compte 1466610 professeurs sous contrat pour les enseignements de base, moyen supérieur et supérieur, chiffre qui montre la disproportion entre le nombre d'élèves et le nombre d'enseignants. Peu de maîtres pour 
l'enseignement et beaucoup pour les fonctions administratives, sans responsabilité de classe.

13 Les enseignants du secteur public, dans les établissements non autonomes, sont affiliés au Syndicat national des personnels de l'éducation. Avec environ 1,5 million d'inscrits (enseignants et personnel administratif du SEM), c'est le syndicat le plus important d'Amérique latine : l'affiliation est automatique et obligatoire pour tout enseignant ou personnel de l'enseignement de base public dans le pays. La structure du syndicat est pyramidale et, s'il existe bien dans l'organigramme un comité exécutif général, on trouve à la tête de l'organisation une direction qui possède un grand pouvoir politique et de contrôle, à l'intérieur du secrétariat à l'Éducation publique lui-même et dans le pays.

\section{Entre un déficit éducatif et une offre de travail insuffisante}

Examinons quelques indicateurs de rendement scolaire pour l'année 2010-2011, afin d'illustrer le déficit du pays en matière d'éducation.

Par ailleurs, on estime que 9,9\% de la population âgée de 25 à 64 ans a été scolarisée.

En ce qui concerne les évaluations internationales PISA, le Mexique a été systématiquement valorisé dans les hautes sphères du pouvoir. Le dernier rapport de gouvernement du président de la République souligne les niveaux élevés atteints par le Mexique en lecture, lors de la dernière évaluation.

Le panorama d'ensemble reste décourageant et bien que les données mentionnées cidessus puissent, dans certains cas, indiquer des pourcentages faibles ou modérés, comme dans le cas de l'abandon, le constat de déficit en éducation est assez indiscutable.

\begin{tabular}{|l|c|c|c|}
\hline \multirow{2}{*}{$\begin{array}{c}\text { Indicateurs } \\
\text { de rendement scolaire }\end{array}$} & \multicolumn{2}{|c|}{ Enseignement de base } & \multirow{2}{*}{$\begin{array}{c}\text { Enseignement moyen } \\
\text { supérieur }\end{array}$} \\
\cline { 2 - 3 } & Primaire & Secondaire & $63,3 \%$ \\
\hline Efficience finale & $95 \%$ & $82,9 \%$ & $14,5 \%$ \\
\hline Abandon & $8 \%$ & $5,6 \%$ & $33,2 \%$ \\
\hline Échec & $3,4 \%$ & $15 \%$ & \\
\hline
\end{tabular}

L'Institut national d'éducation des adultes, organisme officiel, a établi dans un rapport de 2010 que le déficit scolaire au Mexique touchait 33,2\% des 77 millions de personnes que représente la population âgée de 15 ans et plus. Ce déficit se traduit de la façon suivante : près de six millions d'analphabètes, presque 10 millions de personnes qui n'ont pas terminé le cycle primaire et 17,5 millions le cycle secondaire (INEE, 2009).

On observe une autre manifestation de ce déficit dans l'analyse de la population de jeunes qui ne travaillent pas et n'étudient pas - population désignée dans le monde ibéroaméricain par l'expression un peu péjorative «ni-ni ». Alors qu'en 2005, 10,5\% de la tranche d'âge des 14-34 ans se trouvaient dans cette situation, ce pourcentage est monté à $13,12 \%$ en 2010 .

20 Ces données montrent que, malgré l'énorme et complexe structure du système éducatif mexicain, il ne semble pas, actuellement, qu'une attention suffisante soit portée à la nécessité d'une éducation de base et d'une éducation supérieure de la population. Le discours politique public attribue la responsabilité de la situation au mauvais fonctionnement du système, et plus particulièrement aux enseignants. C'est pourquoi les principales stratégies prévues dans les dernières réformes éducatives visent à 
modifier la formation, la contractualisation, la promotion et la permanence du corps enseignant. Le plan d'action pour la réforme du statut contractuel et des conditions de travail des enseignants s'intitule " Alliance pour la qualité de l'éducation ». D'autre part, on observe avec attention que ces réformes prévoient d'importants investissements dans l'infrastructure matérielle et informatique.

Pour tenter de pallier le déficit éducatif signalé par les indicateurs de rendement scolaire et par l'augmentation du pourcentage de jeunes sans études ni travail, le pouvoir fédéral a opté pour des stratégies compensatoires, principalement des programmes de bourses à tous les niveaux d'éducation.

\section{Le financement de l'éducation, les principaux programmes}

Le dernier rapport de gouvernement prévoit un budget global de 909,021 millions de pesos pour l'éducation (plus de 67 millions US\$).

Pour l'année scolaire 2010-2011, la dépense publique moyenne par élève a été de 14000 pesos (1 036 US\$), qui se répartissent selon les niveaux en 12000 pesos (888 US\$) par élève dans le primaire, 19000 pesos (1 372 US\$) dans le secondaire, 27000 pesos (1 949 US\$) dans le cycle du baccalauréat et plus de 60000 pesos (4 331,3 US\$) pour l'enseignement supérieur.

ne part importante du budget général de l'éducation a été destinée à l'infrastructure (réhabilitation ou amélioration) et à l'équipement, y compris à la formation du personnel qui y est rattaché.

Une autre rubrique concerne le renforcement de la décentralisation de l'éducation et consiste en programmes d'appui aux systèmes éducatifs des États.

Plusieurs programmes ont été financés pour remédier au déficit éducatif: un plan d'éducation tout au long de la vie, des postes d'enseignants dans les régions rurales, l'ouverture de services de formation au travail et un programme de formation aux compétences.

27 Le programme «Alliance pour la qualité de l'éducation » a été le plus soutenu, avec la mise en place des récentes réformes comprenant de nouveaux programmes d'enseignement et de nouvelles politiques de contractualisation, de promotion, de formation et d'évaluation des enseignants. C'est donc le programme phare du moment qui recueille la plus grande part du financement.

Des bourses sont attribuées à tous les niveaux de l'enseignement. Pour l'année 2010-2011, on compte plus de six millions d'élèves et étudiants boursiers, du primaire au troisième cycle universitaire. Néanmoins, avec une population scolaire de plus de 34 millions d'élèves et étudiants et plus de 77 millions de personnes en déficit scolaire, cette stratégie compensatoire semble loin d'être suffisante.

Autre stratégie compensatoire : le programme "Deuxième chance ", qui comporte des bourses scolaires dont ont bénéficié plus de cinq millions d'élèves, sans que l'on puisse savoir s'ils font partie des six millions déjà évoqués ou s'ils s'y ajoutent.

Dans cette rubrique figurent les aides au Conseil national de développement éducatif dont l'action porte principalement sur les zones marginalisées du pays, zones rurales et indigènes. 


\section{La recherche en éducation : les étapes de sa structuration}

31 Le système éducatif mexicain s'est développé dans les quatre dernières décennies en partie sous l'influence de la recherche en éducation, qui s'est institutionnalisée dans le pays en tant que pratique universitaire et outil d'intervention. On a commencé à promouvoir et à soutenir la mise en place de départements de recherche en éducation dans les années soixante-dix. Peu à peu, par périodes, l'impact de ces recherches sur le système éducatif a augmenté, à tel point que plusieurs chercheurs en éducation ont fait partie des instances gouvernementales pour la politique éducative, surtout dans les années quatre-vingt-dix et au début du XXI ${ }^{e}$ siècle.

Depuis que le Mexique a intégré l'OCDE dans les années quatre-vingt-dix, les politiques éducatives internationales régissent de façon plus directe les destinées de la politique nationale, ce qui limite l'intervention ou la participation de la recherche en éducation. On fait appel aux chercheurs à certains moments précis, pour donner des orientations, du contenu ou mettre en œuvre les grandes lignes, les programmes et les stratégies déjà définis au niveau international.

\section{Les premières années}

33 Le premier organisme spécialisé dans la recherche en éducation, le Centre d'études éducatives, a été créé au début des années soixante par Pablo Latapi, alors prêtre catholique. Ce centre, et la personne de Latapi, ont acquis une large reconnaissance dans le milieu universitaire et le milieu de la politique éducative. Latapi lui-même était connu du public averti dans ce domaine.

Dans les années soixante, le gouvernement fédéral et les instances académiques autonomes, telles que les universités, ont commencé à institutionnaliser la recherche en éducation au Mexique, dans un contexte de large investissement financier dans l'éducation, d'une nette augmentation du taux de scolarisation, du développement des infrastructures à tous les niveaux d'enseignement et d'une diversification des options éducatives. On a alors créé de nombreux centres de recherche dont, pour la première fois, la tâche principale fut de réaliser une recherche en éducation de façon professionnelle. On a défini et mis en place des contrats professionnels de chercheurs et l'on a produit les premières publications et les premiers programmes de formation spécialisés pour des chercheurs en éducation au Mexique.

La recherche en éducation s'est mise en place dans des organismes spécifiques, spécialisés par niveaux d'enseignement ou par thèmes : enseignement primaire et communautaire au Centre d'études éducatives, programmes d'enseignement pour le primaire et l'enseignement technologique dans le Département de recherches en éducation, systématisation de l'enseignement et formation des enseignants de niveau supérieur au Centre de recherches et de services éducatifs, pour ne citer que les principaux.

36 L'incitation à la création de ces organismes a été très marquée au centre du pays, dans la capitale fédérale et ses environs, mais plus timide dans les autres États de la république fédérale. 


\section{La consolidation de la recherche en éducation comme champ d'études}

Dans les années soixante, l'infrastructure institutionnelle pour réaliser des recherches en éducation a été établie. Puis, au cours des années quatre-vingt, alors qu'une crise économique générale affectait le milieu de l'éducation en bloquant son évolution pour toute une décennie, les chercheurs ont mûri leurs propositions d'analyse, créé des groupes, formé des disciples, défini des cadres académiques et des espaces d'intervention pour l'exercice de leur travail.

Pour donner plus d'élan à ce travail académique, un des leviers consista à organiser le premier congrès national de recherche en éducation. Encore une initiative de Pablo Latapi, à un moment où des universitaires reconnus participaient au gouvernement fédéral et faisaient appel à lui pour appuyer le travail d'impulsion en faveur de la science et de la technologie dans le pays.

Cette première rencontre nationale rendit nécessaire un travail interinstitutionnel entre des chercheurs qui, jusque là, s'identifiaient d'abord à leur institution, et commencèrent à partir de ce moment à se regrouper entre spécialistes s'occupant des mêmes thèmes de recherche - premier pas pour dépasser le cadre de l'institution et contribuer davantage à la constitution d'un champ d'études.

40 Parallèlement, des publications sur les recherches en éducation se développaient en nombre et en solidité, alors que dans le reste du pays on continuait à ouvrir des centres de recherche en éducation, surtout dans les institutions publiques supérieures de recherche.

\section{Organisation en communautés de recherche en éducation}

41 À la fin des années quatre-vingt, on a pu identifier les premières communautés ou réseaux de recherche entre chercheurs et professionnels de l'éducation, comme les enseignants et les étudiants de troisième cycle. On peut citer, par exemple, les réseaux « Didactique des mathématiques » et «Éducation et travail ». Dans ce type d'organisation académique, les membres se regroupaient par intérêt personnel et de façon spontanée autour des thèmes et axes de travail de chaque réseau ou communauté, qui fonctionnaient grâce aux ressources que les différents membres pouvaient apporter de leurs institutions de rattachement respectives.

La production de connaissances dans le domaine de la recherche en éducation commença à dépasser le cadre institutionnel et la logique de travail académique de chaque institution cessa d'être le point de départ du développement des thèmes de recherche. L'échange, l'interaction, la collaboration et la production conjointe et directe entre des acteurs différents permirent de traiter avec efficacité des thèmes émergents, de surmonter les contraintes économiques et de contourner les délais institutionnels d'approbation ou d'avalisation des projets et demandes de recherche.

Pendant les années quatre-vingt-dix, dans le cadre d'une brève amélioration économique qui dura quelques années, et même ensuite dans un scénario de retour de crise, la politique fédérale maintint son soutien au champ de la science et de la technologie, considéré comme un axe de développement économique et social. Les mesures prises 
en ce sens eurent aussi une incidence favorable sur la recherche en éducation. Appui économique, infrastructure et programmes de financement spécifique furent proposés pour des projets de recherche par appels d'offres. On commença à percevoir nettement un regain d'activités dans les institutions académiques en général, et dans le domaine de la recherche en éducation en particulier, l'ensemble étant fortement soutenu par des politiques d'évaluation académique qui furent appliquées au niveau national de façon standardisée.

Les communautés et réseaux de recherche devinrent ainsi un mode de fonctionnement plus dynamique pour répondre au nouveau modèle politique de soutien à la science et à la technologie et de régulation académique.

\section{Le Conseil mexicain de recherche en éducation}

Un second congrès national de recherche en éducation a donné lieu à la création d'un Conseil national de chercheurs. Depuis 1993, ce conseil a regroupé des chercheurs de diverses institutions du pays. Ses membres fondateurs ont été les pionniers de la recherche en éducation au Mexique : ils sont parvenus à mettre en place un congrès bisannuel, une publication semestrielle, la publication de différents travaux académiques et à appuyer et développer diverses actions de recherche sur le système éducatif, par contrat ou convention. Au-delà des actions spécifiques, le Conseil est devenu un acteur important du domaine de l'éducation et un référent pour la politique éducative du pays.

Le conseil regroupe seize domaines thématiques à partir desquels s'organisent les congrès. Dans chacun des domaines, on trouve différents modes d'organisation, en réseau et communauté, selon le choix des membres.

Tout en étant une organisation de chercheurs, le Conseil a qualité pour inviter à ses congrès bisannuels des professeurs et étudiants de tout le pays, ainsi que pour ouvrir des espaces de dialogue et de débats avec les fonctionnaires publics. Ces manifestations sont parvenues à réunir plus de 2500 personnes intéressées par ce champ d'études.

En certaines occasions, le Conseil a pu exprimer une attitude critique par rapport à la politique fédérale, sachant que ses membres peuvent avoir des postures différentes et assumer divers rôles dans ce domaine.

Avec plus de trois cents membres, il s'agit d'une association spécifique, qui par ses fonctions et actions, a un statut particulier dans l'orbite sud-américaine.

Cette brève présentation a proposé une vue d'ensemble de l'éducation au Mexique et, plus particulièrement de la situation de la recherche en éducation dans ce pays. Il ne faut cependant pas omettre de signaler le risque important que court le secteur public de l'éducation, avec la situation de violence que connaît actuellement le pays. La criminalité touche désormais les écoles sous forme de racket des enseignants. Certaines écoles de l'État de Guerrero ont fermé leurs classes et l'institution toute entière a cessé le travail jusqu'à ce que des garanties soient prises pour la sécurité du travail scolaire (Jornada, 30 septembre 2011). Bien qu'il s'agisse d'un délit qui relève de la justice, il peut être utile de l'évoquer comme un élément du panorama présenté car il affecte et entache gravement l'éducation et le pays tout entier. 


\section{BIBLIOGRAPHIE}

CALDERÓN, presidente, 2007-2012 (2011) : V Informe de Gobierno. Gobierno Federal, México. Instituto Nacional de Evaluación Educativa (2011) : Panorama Educativo de México. Indicadores del Sistema Educativo Nacional, 2009. Educación Media Superior. INEE : México.

LA JORNALADA (2011) : Periódico La jornada, México. 30 septembre 2011.

LOYO A. (2010) : «El papel histórico del SNTE », document électronique, non publié.

NIETO D.M. (2009) : Análisis de las politicas para maestros de educación básica en México. México : UI/ OCDE.

www.snte.org.mx, [consulté le 25 septembre 2011].

\section{NOTES}

1. Article traduit par Odile Luginbühl.

\section{INDEX}

Index géographique : Mexique

Mots-clés : droit à l'éducation, enseignement public, recherche en éducation, scolarisation

Keywords : right to education, public education, educational research, schooling

Palabras claves : derecho a la educación, enseñanza pública, investigación en educación, escolarización

\section{AUTEUR}

\section{NORMA GEORGINA GUTIÉRREZ SERRANO}

Chercheuse (Investigadora Titular) à l'Universidad Nacional Autónoma de México, Centre régional de recherches multidisciplinaires, Mexique. 